\title{
KURZBESPRECHUNGEN
}

\author{
OsWALD ITEN
}

Economic Pressures on Traditional Societies

A Case Study of Southeastern Nuba Economy in the Modern Sudan

European University Papers, Series XXIX, Vol. 8, Peter Lang, Bern/Frankfurt/Las Vegas, 1979, 163 S.

Die Nuba im südlichen Sudan sind nicht nur wegen Besonderheiten ihrer Gesellschaftsstruktur Gegenstand einer reichen ethnologischen Forschung, sondern wegen gewisser exotischer Aspekte ihres Brauchtums (Nacktheit, blutige Wettkämpfe, Körperbemalung) etwa durch die Bildbände Leni Riefenstahls auch einer breiteren Offentlichkeit bekannt. Itens Studie über ihre Wirtschaft ist nicht nur ein Beitrag zur wirtschaftlichen Ethnologie, sondern auch zur aktuellen entwicklungstheoretischen Diskussion. Der Verfasser zeigt nämlich unter erfreulicher Beschränkung auf das Wesentliche in einer sorgfältigen Analyse ihrer Produktionsweise und Handelsbeziehungen, daß es ihnen mit ,traditionellen" Produktionsmethoden nicht nur gelingt, ihre Subsistenz zu sichern, sondern auch noch einen erheblichen Uberschuß zu erwirtschaften, wobei sie für diesen allerdings aufgrund der Monopolisierung des Handels durch arabische Händler einen eher unbefriedigenden Ertrag erhalten. In nüchterner Sprache und gut belegt, erledigt Iten dabei nebenbei eine ganze Reihe von theoretischen Glaubenssätzen (über die Unfähigkeit klassenloser Gesellschaften, Mehrwert zu produzieren, über die Primitivität von Okonomien, deren Technologie nicht über den Grabstock hinausgelangt ist usw.). Im sudanesischen Kontext sind die Nuba reich. Sinnvolle Entwicklungspolitik müßte hier ansetzen und gezielte Verbesserungen (in der Wasserversorgung, in der Vermarktung) anbringen. Statt dessen laufen die Planungen der sudanesischen Regierung und ausländischer Förderorganisationen auf ,, Modernisierungen“ hinaus, von denen mit Sicherheit vorausgesagt werden kann, daß sie bestenfalls ineffektiv sind, schlimmstenfalls zur wirtschaftlichen wie kulturellen Verarmung der Nuba führen werden. Nicht zu unterschätzen sind dabei die Vorurteile der arabo-islamischen Bürokratie, denen die eingangs erwähnten Aspekte der Kultur der Nuba, die sie zur Touristenattraktion gemacht hat, als Schande für den Sudan erscheinen. Der Islam, der im Vorderen Orient die Rolle einer identitätswahrenden Ideologie gegen Verwestlichung spielt, erweist sich in diesem Kontext (wie auch in anderen afrikanischen Gesellschaften) als modernisierende, kultur-imperialistische Ideologie.

Brun-Otto Bryde

\section{Peter Meyns}

\section{Befreiung und nationaler Wiederaufbau von Moçambique}

Studien zu Politik und Wirtschaft 1960-1978, Institut für Afrika-Kunde Hamburg, Nr. 18, 1979, 279 S., 14,- DM.

Dieser Band vereint drei Aufsätze des Autors zur politischen Entwicklung (Der Kampf für nationale Unabhängigkeit der FRELIMO [seit 1960] sowie der politischen Struktur und Entwicklung des völkerrechtlich unabhängigenStaates [seit 1975]), zur Wirtschaft (Vom kolonialen Erbe bis zum Aufbau einer nationalen Wirtschaft) sowie über die ländliche Entwicklungsstrategie in dieser ehemaligen portugiesischen Kolonie und heutigen Volksrepu- 